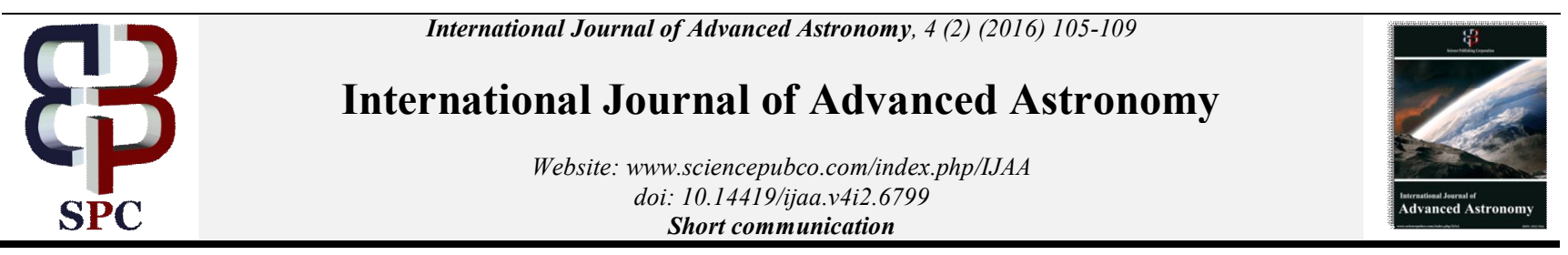

\title{
Final unification with three gravitational constants associated with nuclear, electromagnetic and gravitational interactions
}

\author{
Seshavatharam.U.V.S ${ }^{1 *}$, S. Lakshminarayana ${ }^{2}$ \\ ${ }^{1}$ Honorary faculty, I-SERVE, Alakapuri, Hyderabad-35, Telangana, INDIA \\ ${ }^{2}$ Dept. of Nuclear Physics, Andhra University, Visakhapatnam-03,AP, INDIA \\ *Corresponding author E-mail: seshavatharam.uvs@gmail.com
}

\begin{abstract}
By introducing two large pseudo gravitational constants assumed to be associated with strong and electromagnetic interactions, we make an attempt to combine the old Abdus Salam's 'strong gravity' concept with 'Newtonian gravity' and try to understand the constructional features of nuclei, atoms and neutron stars in a unified approach. From the known elementary atomic and nuclear physical constants, estimated magnitude of the Newtonian gravitational constant is $(6.66$ to 6.70$) \times 10^{-11} \mathrm{~m}^{3} / \mathrm{kg} / \mathrm{sec}^{2}$. Finally, by eliminating the proposed two pseudo gravitational constants, we inter-related the Newtonian gravitational constant, Fermi's weak coupling constant and Strong coupling constant, in a generalized approach.
\end{abstract}

Keywords: Final Unification; Gravitational Constants Associated with Strong and Electromagnetic Interactions.

\section{Introduction}

Even though 'String theory' models' [1], [2] are having a strong mathematical back ground and sound physical basis, they are failing in implementing the Newtonian gravitational constant in atomic and nuclear physics and thus seem to fail in developing a 'workable' model of final unification. It clearly indicates our lack of understanding and uncertain assumptions on which our current physics is being built up. The main issue is: to understand the basics of final unification from hidden, unknown and un-identified physics! Based on the old and ignored scientific assumption put forward by Nobel laureate Abdus Salam [3], we propose two large pseudo gravitational constants assumed to be associated with strong and electromagnetic interactions $[4,5,6]$. With them, currently believed generalized physical constants like, protonelectron mass ratio, neutron life time, weak coupling constant, strong coupling constant, nuclear charge radius, root mean square radius of proton, Planck's constant, Bohr radius of hydrogen atom, molar mass constant, Avogadro number and Newtonian gravitational constant etc and concepts like nuclear binding energy, nuclear stability, nuclear charge radii and atomic radii can be reviewed in a unified approach. In addition, neutron star mass and radius can be understood with the ratio of nuclear to Newtonian gravitational constants.

\section{Two basic assumptions of final unification}

Assumption-1: Magnitude of the gravitational constant associated with the electromagnetic interaction is,

$$
\mathrm{G}_{\mathrm{e}} \cong(2.375 \pm 0.002) \times 10^{37} \mathrm{~m}^{3} \mathrm{~kg}^{-1} \mathrm{sec}^{-2}
$$

Assumption-2: Magnitude of the gravitational constant associated with the strong interaction is

$\mathrm{G}_{\mathrm{S}} \cong(3.328 \pm 0.002) \times 10^{28} \mathrm{~m}^{3} \mathrm{~kg}^{-1} \mathrm{sec}^{-2}$

Note: We chose $\left(\mathrm{G}_{\mathrm{e}}, \mathrm{G}_{\mathrm{S}}\right)$ in such a way that,

$$
\begin{aligned}
& \frac{\mathrm{m}_{\mathrm{p}}}{\mathrm{m}_{\mathrm{e}}} \cong\left(\frac{\mathrm{G}_{\mathrm{s}} \mathrm{m}_{\mathrm{p}}^{2}}{\hbar \mathrm{c}}\right)\left(\frac{\mathrm{G}_{\mathrm{e}} \mathrm{m}_{\mathrm{e}}^{2}}{\hbar \mathrm{c}}\right) \\
& \left(\frac{\mathrm{G}_{\mathrm{s}} \mathrm{m}_{\mathrm{p}} \mathrm{m}_{\mathrm{e}}}{\hbar \mathrm{c}}\right) \cong\left(\frac{\hbar \mathrm{c}}{\mathrm{G}_{\mathrm{e}} \mathrm{m}_{\mathrm{e}}^{2}}\right)
\end{aligned}
$$

\section{Relation between $\mathrm{m}_{\mathrm{p}}$ and $\mathrm{m}_{\mathrm{e}}$}

Based on the Planck mass, $\mathrm{M}_{\mathrm{pl}} \cong \sqrt{\hbar \mathrm{c} / \mathrm{G}_{\mathrm{N}}}$

$\mathrm{m}_{\mathrm{p}} \cong\left(\frac{\mathrm{G}_{\mathrm{N}}}{\mathrm{G}_{\mathrm{e}}}\right)^{1 / 6} \sqrt{\mathrm{M}_{\mathrm{pl}} \mathrm{m}_{\mathrm{e}}}$

If nuclear Planck mass is defined as,

$$
\begin{aligned}
& \mathrm{m}_{\mathrm{npl}} \cong \sqrt{\hbar \mathrm{c} / \mathrm{G}_{\mathrm{s}}} \approx 546.7 \mathrm{MeV} / \mathrm{c}^{2} \\
& \mathrm{~m}_{\mathrm{e}} \cong\left(\frac{\mathrm{m}_{\mathrm{p}}^{5} \mathrm{~m}_{\mathrm{npl}}^{2}}{\mathrm{M}_{\mathrm{pl}}}\right)^{1 / 6} \cong\left(\left(\frac{\mathrm{G}_{\mathrm{N}}}{\mathrm{G}_{\mathrm{s}}}\right) \mathrm{m}_{\mathrm{npl}}^{2} \mathrm{~m}_{\mathrm{p}}^{10}\right)^{1 / 12}
\end{aligned}
$$


$\mathrm{m}_{\mathrm{p}} \cong\left(\frac{\mathrm{m}_{\mathrm{e}}^{6} \mathrm{M}_{\mathrm{pl}}}{\mathrm{m}_{\mathrm{npl}}^{2}}\right)^{1 / 5} \cong\left(\left(\frac{\mathrm{G}_{\mathrm{s}}}{\mathrm{G}_{\mathrm{N}}}\right) \frac{\mathrm{m}_{\mathrm{e}}^{12}}{\mathrm{~m}_{\mathrm{npl}}^{2}}\right)^{1 / 10}$

$\hbar \cong\left(\frac{\mathrm{G}_{\mathrm{s}}}{\mathrm{G}_{\mathrm{N}}}\right)\left(\frac{\mathrm{m}_{\mathrm{e}}}{\mathrm{m}_{\mathrm{p}}}\right)^{10}\left(\frac{\mathrm{G}_{\mathrm{s}} \mathrm{m}_{\mathrm{e}}^{2}}{\mathrm{c}}\right)$

\section{To fix the magnitudes of $\mathrm{G}_{\mathrm{s}}, \mathrm{G}_{\mathrm{e}}$ and $\mathrm{G}_{\mathrm{N}}$}

It is possible to obtain the following relation.

$\mathrm{h} \cong \sqrt{\frac{\mathrm{m}_{\mathrm{p}}}{\mathrm{m}_{\mathrm{e}}}} \sqrt{\left(\frac{\mathrm{G}_{\mathrm{s}} \mathrm{m}_{\mathrm{p}}^{2}}{\mathrm{c}}\right)\left(\frac{\mathrm{e}^{2}}{4 \pi \varepsilon_{0} \mathrm{c}}\right)}$

Based on this relation [3],

$$
\begin{aligned}
& \mathrm{G}_{\mathrm{s}} \cong \frac{4 \pi \varepsilon_{0} \mathrm{~h}^{2} \mathrm{c}^{2} \mathrm{~m}_{\mathrm{e}}}{\mathrm{e}^{2} \mathrm{~m}_{\mathrm{p}}^{3}} \cong 3.329560807 \times 10^{28} \frac{\mathrm{m}^{3}}{\mathrm{~kg} \cdot \mathrm{sec}^{2}} \\
& \mathrm{G}_{\mathrm{e}} \cong\left(\frac{\mathrm{e}^{2} \mathrm{~m}_{\mathrm{p}}^{2}}{16 \pi^{3} \varepsilon_{0} \mathrm{~m}_{\mathrm{e}}^{4}}\right) \cong 2.374335471 \times 10^{37} \frac{\mathrm{m}^{3}}{\mathrm{~kg} \cdot \mathrm{sec}^{2}} \\
& \mathrm{G}_{\mathrm{N}} \cong\left(\frac{\mathrm{m}_{\mathrm{e}}}{\mathrm{m}_{\mathrm{p}}}\right)^{14}\left(\frac{4 \pi \varepsilon_{0}}{\mathrm{e}^{2}}\right)^{2}\left(\frac{2 \pi \mathrm{h}^{3} \mathrm{c}^{3}}{\mathrm{~m}_{\mathrm{p}}^{2}}\right) \\
& \cong 6.679856051 \times 10^{-11} \mathrm{~m}^{3} \mathrm{~kg}^{-1} \mathrm{sec}^{-2}
\end{aligned}
$$

\section{To fit neutron life time and strong coupling constant}

Let, $t_{n}$ be the life time of neutron. Quantitatively it is possible to show that [7],

$$
\frac{\left(\mathrm{m}_{\mathrm{n}}-\mathrm{m}_{\mathrm{p}}\right)}{\mathrm{m}_{\mathrm{n}}} \cong \sqrt{\frac{\mathrm{G}_{\mathrm{e}}}{\mathrm{G}_{\mathrm{N}}}}\left(\frac{\mathrm{G}_{\mathrm{s}} \mathrm{m}_{\mathrm{n}}}{\mathrm{c}^{3} \mathrm{t}_{\mathrm{n}}}\right)
$$

where $\sqrt{\frac{\mathrm{G}_{\mathrm{e}}}{\mathrm{G}_{\mathrm{N}}}} \cong 5.96 \times 10^{23} \approx$ Avogadro number.

$$
\mathrm{t}_{\mathrm{n}} \cong \sqrt{\frac{\mathrm{G}_{\mathrm{e}}}{\mathrm{G}_{\mathrm{N}}}}\left(\frac{\mathrm{G}_{\mathrm{s}} \mathrm{m}_{\mathrm{n}}^{2}}{\left(\mathrm{~m}_{\mathrm{n}}-\mathrm{m}_{\mathrm{p}}\right) \mathrm{c}^{3}}\right) \approx 896 \mathrm{sec}
$$

With reference to the Weak coupling constant $G_{F}$ and the proposed $G_{S}$,

$$
G_{N} \cong\left(\frac{m_{e}}{m_{p}}\right)^{7 / 2} \frac{\sqrt{G_{s} G_{F}}}{2 t_{n}\left(m_{n}-m_{p}\right) c}
$$

If one is willing to define the strong coupling constant [7] as,

$$
\alpha_{\mathrm{S}} \cong\left(\frac{\hbar \mathrm{c}}{\mathrm{G}_{\mathrm{S}} \mathrm{m}_{\mathrm{p}}^{2}}\right)^{2},
$$

$\mathrm{t}_{\mathrm{n}} \cong \sqrt{\frac{\mathrm{G}_{\mathrm{e}}}{\mathrm{G}_{\mathrm{N}}}} \sqrt{\frac{1}{\alpha_{\mathrm{s}}}}\left(\frac{\hbar}{\left(\mathrm{m}_{\mathrm{n}}-\mathrm{m}_{\mathrm{p}}\right) \mathrm{c}^{2}}\right) \cong \frac{303.42 \mathrm{sec}}{\sqrt{\alpha_{\mathrm{s}}}}$

If, $\alpha_{\mathrm{S}} \cong 0.1185 \pm 0.0006, \mathrm{t}_{\mathrm{n}} \cong 881.422 \mathrm{sec}$

$$
\mathrm{G}_{\mathrm{N}} \cong \frac{1}{\alpha_{\mathrm{S}}}\left(\frac{\hbar}{\mathrm{t}_{\mathrm{n}}\left(\mathrm{m}_{\mathrm{n}}-\mathrm{m}_{\mathrm{p}}\right) \mathrm{c}^{2}}\right)^{2} \mathrm{G}_{\mathrm{e}} \cong 6.6991 \times 10^{-11} \frac{\mathrm{m}^{3}}{\mathrm{~kg} \cdot \mathrm{sec}^{2}}
$$

Where, $\quad \alpha_{\mathrm{S}} \cong 0.1185 \pm 0.0006, \mathrm{t}_{\mathrm{n}} \cong(880.3 \pm 1.1) \mathrm{sec}$.

\section{To fit the nuclear charge radius and root mean square radius of proton}

Nuclear charge radius can be expressed with the following relation

$R_{0} \cong \frac{2 G_{s} m_{p}}{c^{2}} \cong 1.24 \times 10^{-15} \mathrm{~m}$

Root mean square radius of proton [7] can be expressed with the following relation.

$R_{p} \cong \frac{\sqrt{2} G_{s} m_{p}}{c^{2}} \approx 0.876 \times 10^{-15} \mathrm{~m}$

Based on relations (16) and (17),

$G_{N} \cong\left(\frac{m_{e}}{m_{p}}\right)^{12}\left[\left(\frac{c^{3} R_{0}^{2}}{4 \hbar}\right)\right.$ Or $\left.\left(\frac{c^{3} R_{p}^{2}}{2 \hbar}\right)\right]$

\section{Nuclear binding energy close to stable mass numbers.}

It is noticed that,

$$
\left.\begin{array}{l}
-\left(\frac{3}{5}\left[e^{2} / 4 \pi \varepsilon_{0} R_{p}\right]\right) \cong-0.986 \mathrm{MeV} \\
-\left(\frac{3}{5}\left[G_{s} m_{p}^{2} / R_{p}\right]\right) \cong-398.0 \mathrm{MeV}
\end{array}\right\}
$$

Seem to represent the respective self-binding energies. Then for $(Z \geq 5)$, nuclear binding energy [8], [9] close to stable mass numbers can be expressed with,

$$
\begin{aligned}
& (B E)_{A s} \cong-\left[Z-\left(2-\sqrt{\frac{Z}{30}}\right)\right] \sqrt{\left(\frac{3}{5} \frac{e^{2}}{4 \pi \varepsilon_{0} R_{p}}\right)\left(\frac{3}{5} \frac{G_{s} m_{p}^{2}}{R_{p}}\right)} \\
& \cong-\left[Z-\left(2-\sqrt{\frac{Z}{30}}\right)\right] \times(19.75 \text { to } 19.8) \mathrm{MeV}
\end{aligned}
$$

We are working on refining the ad-hoc expression $[Z-(2-\sqrt{Z / 30})]$. See column-2 of table-1 prepared with 19.75 MeV. With this binding energy constant stable mass number $A_{s}$ can be estimated with,

$A_{s} \approx X+\left(Y^{2}+Y\right)$ 
where, $X \approx\left(Z+\sqrt{\frac{Z}{30}}-2\right)\left(\frac{19.75 \mathrm{MeV}}{8.8 \mathrm{MeV}}\right)$

and $Y \approx\left[(X-2 Z)^{2} / Z\right]$

Considering $8.8 \mathrm{MeV}$ as the maximum binding energy per nucleon, $X$ can be referred to the lowest possible imaginary stable mass number and $\left(A_{s}-X\right) \approx\left(Y^{2}+Y\right)$

Corrections in estimated $A_{s}$ :

If $Z$ is even and estimated $A_{S}$ is even,

Corrected $A_{s}$ range $\cong\left\{\right.$ Estimated $\left.A_{s} \pm 2\right\}$

If $Z$ is even and estimated $A_{s}$ is odd,

Corrected $A_{s}$ range $\cong\left\{\left(\right.\right.$ Estimated $\left.\left.A_{s}-1\right) \pm 2\right\}$

If $Z$ is odd and estimated $A_{s}$ is odd,

Corrected $A_{s}$ range $\cong\left\{\right.$ Estimated $\left.A_{s} \pm 2\right\}$

If $Z$ is odd and estimated $A_{S}$ is even,

Corrected $A_{s}$ range $\cong\left\{\left(\right.\right.$ Estimated $\left.\left.A_{s}-1\right) \pm 2\right\}$

Table 1: To Estimate Medium, Heavy and Super Heavy Atomic Nuclides and Their Binding Energy

\begin{tabular}{|c|c|c|c|}
\hline $\begin{array}{l}\text { Proton } \\
\text { number }\end{array}$ & $\begin{array}{l}\text { Estimated Binding } \\
\text { energy close to } \\
\mathrm{A}_{\mathrm{s}}(\mathrm{MeV})\end{array}$ & $\begin{array}{l}\text { Estimated } \\
\text { stable mass num- } \\
\text { ber with even-odd } \\
\text { correction }\end{array}$ & $\begin{array}{l}\text { Actual (stable } \\
\text { and long living) } \\
\text { isotopes }\end{array}$ \\
\hline 21 & 391.8 & $45 \pm 2$ & 45 \\
\hline 25 & 472.3 & $53 \pm 2$ & 55 \\
\hline 31 & 592.8 & $69 \pm 2$ & 69,71 \\
\hline 35 & 673.1 & $79 \pm 2$ & 79,81 \\
\hline 41 & 793.3 & $93 \pm 2$ & 93 \\
\hline 47 & 913.5 & $109 \pm 2$ & 107,109 \\
\hline 51 & 993.5 & $119 \pm 2$ & 121,123 \\
\hline 55 & 1073.5 & $131 \pm 2$ & 133 \\
\hline 59 & 1153.4 & $141 \pm 2$ & 141 \\
\hline 60 & 1173.4 & $144 \pm 2$ & $\begin{array}{l}142,144,146, \\
143,145, \\
148,150\end{array}$ \\
\hline 65 & 1273.3 & $159 \pm 2$ & 159 \\
\hline 69 & 1353.2 & $169 \pm 2$ & 169 \\
\hline 75 & 1473.0 & $187 \pm 2$ & 187,185 \\
\hline 81 & 1592.7 & $205 \pm 2$ & 205,203 \\
\hline 86 & 1692.4 & $220 \pm 2$ & 222 \\
\hline 92 & 1812.1 & $238 \pm 2$ & 238,235 \\
\hline 100 & 1971.6 & $262 \pm 2$ & 257 \\
\hline
\end{tabular}

In this table, estimated stable mass numbers can be understood with the following relation.

$A_{s} \approx 2 Z+k(2 Z)^{2} \approx Z+0.0064 Z^{2}$

where, $k \cong\left(\frac{G_{s} m_{p} m_{e}}{\hbar c}\right) \cong\left(\frac{\hbar c}{G_{e} m_{e}^{2}}\right) \cong 1.605 \times 10^{-3}$,

Quantitatively this relation can be compared with the computationally proposed relation (8) of reference [8] which takes the following form.

$N_{s} \cong 0.968051 Z+0.00658803 Z^{2}$ where $N_{s}$ is the neutron number of a nucleus with atomic number $\mathrm{Z}$ on the line of beta stability. Based on 'mass number', relation (27) can also be expressed in the following form.

$Z \approx \frac{\sqrt{4 k A+1}-1}{4 k}$

where $A$ is any mass number. This relation (28) can be compared with existing stability relation,

$$
Z \approx \frac{A}{2+\left(a_{c} / 2 a_{a}\right) A^{2 / 3}}
$$

where $\left(a_{c} / 2 a_{a}\right) \cong 0.0157$. Keeping 'workability' point of view and 'final unification' point of view, proposed two assumptions, can be recommended for further research and analysis.

\section{To fit Fermi's weak coupling constant and Newtonian gravitational constant}

To a great surprise, it is noticed that [7],

$G_{F} \cong\left(\frac{m_{e}}{m_{p}}\right)^{2} \hbar c R_{0}^{2}$

From above relations,

$$
\begin{aligned}
& G_{F} \cong\left(\frac{m_{e}}{m_{p}}\right)^{2}\left(\frac{4 G_{s}^{2} m_{p}^{2} \hbar}{c^{3}}\right) \cong\left(\frac{4 G_{s}^{2} m_{e}^{2} \hbar}{c^{3}}\right) \\
& \cong 1.44 \times 10^{-62} \mathrm{~J} \cdot \mathrm{m}^{3} \\
& G_{N} \cong\left(\frac{m_{e}}{m_{p}}\right)^{10}\left(\frac{G_{F} c^{2}}{4 \hbar^{2}}\right) \cong 6.66 \times 10^{-11} \frac{\mathrm{m}^{3}}{\mathrm{~kg} \cdot \mathrm{sec}^{2}} \\
& \text { where, } G_{F} \cong 1.1663787 \times 10^{-5}(\hbar c)^{3} \mathrm{GeV}^{-2}
\end{aligned}
$$

\section{Mass and radius of neutron star}

Let $\left(M_{N S}, \mathrm{R}_{\mathrm{NS}}\right)$ represent mass and radius of neutron star [10], [11] respectively. It is noticed that,

$$
\begin{gathered}
\frac{G_{N} M_{N S} m_{n}}{\hbar c} \approx \sqrt{\frac{G_{s}}{G_{N}}} \\
M_{N S} \approx \sqrt{\frac{G_{S}}{G_{N}}}\left(\frac{\hbar c}{G_{N} m_{n}}\right) \approx 3.17 \text { Solar mass }
\end{gathered}
$$

$\frac{R_{N S}}{\left(\sqrt{G_{N} \hbar / c^{3}}\right)} \approx \frac{G_{S}}{G_{N}}$

$$
\begin{aligned}
& R_{N S} \approx\left(\frac{G_{S}}{G_{N}}\right) \sqrt{\frac{\hbar G_{N}}{c^{3}}} \approx \sqrt{\frac{G_{s}}{G_{N}}} \sqrt{\frac{\hbar G_{s}}{c^{3}}} \approx 8.1 \mathrm{~km} \\
& \rightarrow \frac{R_{N S}}{\sqrt{\hbar G_{s} / c^{3}}} \simeq \sqrt{\frac{G_{s}}{G_{N}}}
\end{aligned}
$$




\section{0. 'System of units' independent Avogadro number and Molar mass unit}

If, atoms as a whole believed to exhibit electromagnetic interaction, then both molar mass constant and Avogadro number can be understood with:

$$
G_{e}\left(m_{\text {atom }}\right)^{2} \cong G_{N}\left(M_{\text {mole }}\right)^{2}
$$

where, $m_{\text {atom }}$ is the unified atomic mass unit and $M_{\text {mole }}$ is the molar mass unit or gram mole. If so,

$\frac{M_{\text {mole }}}{m_{\text {atom }}} \cong \sqrt{\frac{G_{e}}{G_{N}}} \cong$ Avogadro number

where $\sqrt{\frac{G_{e}}{G_{N}}} \cong 5.96 \times 10^{23}$ and $\left(0.00099>M_{\text {mole }}<0.001\right) \mathrm{kg}$

$M_{\text {mole }} \cong \sqrt{\frac{G_{e}}{G_{N}}} \times m_{\text {atom }}$

\section{To fit and understand the atomic radii}

Considering the geometric mean of the two assumed gravitational constants associated with proton and 'atom as whole', atomic radii can be fitted in the following way. By following the periodic arrangement of atoms and their electronic arrangement, accuracy can be improved.

$R_{\text {atom }} \cong A_{s}^{1 / 3} \sqrt{\left(\frac{2 G_{s} m_{n}}{c^{2}}\right)\left(\frac{2 G_{e} m_{\text {atom }}}{c^{2}}\right)}$

$\cong A_{s}^{1 / 3} * 33.0$ pico.meter

where

$$
\left.\begin{array}{l}
\left(\frac{2 G_{s} m_{n}}{c^{2}}\right) \cong 1.24 \times 10^{-15} \mathrm{~m} ; \\
\left(\frac{2 G_{e} m_{\text {atom }}}{c^{2}}\right) \cong 8.776 \times 10^{-7} \mathrm{~m} ;
\end{array}\right\}
$$

$A_{s}$ is the stable mass number of the atom, $m_{n}$ is the average mass of nucleon and $m_{\text {atom }}$ is the unified atomic mass unit. Note that, this relation resembles the famous relation for estimating nuclear radius [12], [13]. See the following table-2.

Table.2: Estimated Atomic Radii

\begin{tabular}{llll}
\hline $\begin{array}{l}\text { Proton } \\
\text { number }\end{array}$ & $\begin{array}{l}\text { Stable Mass } \\
\text { number }\end{array}$ & $\begin{array}{l}\text { Estimated atomic } \\
\text { radii (pico meter) }\end{array}$ & $\begin{array}{l}\text { Reference data [14] } \\
\text { (pico meter) }\end{array}$ \\
\hline 1 & 1 & 33.0 & 31 \\
6 & 12 & 75.6 & 76 \\
16 & 32 & 104.8 & 105 \\
27 & 57 & 127.0 & 126 \\
28 & 62 & 130.6 & 124 \\
29 & 63 & 131.3 & 132 \\
30 & 66 & 133.4 & 122 \\
40 & 90 & 147.9 & 175 \\
47 & 107 & 156.7 & 145 \\
60 & 142 & 172.2 & 201 \\
70 & 172 & 183.5 & 187 \\
81 & 203 & 193.9 & 145 \\
89 & 227 & 201.3 & 215 \\
92 & 238 & 204.5 & 196 \\
\hline
\end{tabular}

\section{Generalized relations}

Based on the above relations and by eliminating the proposed two pseudo gravitational constants, we pulled-out the following two interesting relations.

Result-1: Proton-electron mass ratio,

$$
\left(\frac{m_{p}}{m_{e}}\right) \cong\left(\frac{G_{F}}{\hbar c R_{p l}^{2}}\right)^{\frac{1}{10}} \cong\left(\frac{G_{F} c^{2}}{4 G_{N} \hbar^{2}}\right)^{\frac{1}{10}}
$$

where $G_{F}$ is the Fermi's weak coupling constant and

$$
R_{p l} \cong \frac{2 G_{N} M_{p l}}{c^{2}} \cong 2 \sqrt{\frac{G_{N} \hbar}{c^{3}}} \text { where } M_{p l} \cong \sqrt{\frac{\hbar c}{G_{N}}}
$$

Result-2: Strong coupling constant,

$$
\alpha_{s} \cong \frac{4 \hbar^{3} m_{e}^{2}}{G_{F} m_{p}^{4} c}
$$

where $\alpha_{s}$ is the strong coupling constant.

Based on these two results,

$$
\begin{aligned}
& \left(\frac{m_{p}}{m_{e}}\right) \cong\left(\frac{\hbar c}{\alpha_{s} G_{N} m_{p}^{2}}\right)^{\frac{1}{12}} \cong\left(\frac{1}{\alpha_{s}}\right)^{\frac{1}{12}}\left(\frac{\hbar c}{G_{N} m_{p}^{2}}\right)^{\frac{1}{12}} \\
& \alpha_{s} G_{F} \cong \frac{4 \hbar^{3} m_{e}^{2}}{m_{p}^{4} c} \\
& G_{F} \cong\left(\frac{1}{\alpha_{s}}\right)\left\{\frac{4 \hbar^{3} m_{e}^{2}}{m_{p}^{4} c}\right\} \cong \frac{1.659031433 \times 10^{-63}}{\alpha_{s}} \mathrm{~J} . \mathrm{m}^{3}
\end{aligned}
$$

With reference to the recommended value of $G_{F}$,

$$
\begin{aligned}
\alpha_{s} & \cong\left\{\frac{4 \hbar^{3} m_{e}^{2}}{m_{p}^{4} c G_{F}}\right\} \cong \frac{1.659031433 \times 10^{-63}}{1.435850984 \times 10^{-62}} \\
& \cong 0.115542236
\end{aligned}
$$

$$
\begin{aligned}
& \alpha_{s} G_{N} \cong\left(\frac{m_{e}}{m_{p}}\right)^{12}\left(\frac{\hbar c}{m_{p}^{2}}\right) \\
& G_{N} \cong\left(\frac{1}{\alpha_{s}}\right)\left\{\left(\frac{m_{e}}{m_{p}}\right)^{12}\left(\frac{\hbar c}{m_{p}^{2}}\right)\right\} \\
& \cong \frac{7.694773265 \times 10^{-12}}{\alpha_{s}} \mathrm{~m}^{3} \cdot \mathrm{kg}^{-1} \mathrm{sec}^{-2}
\end{aligned}
$$

With reference to the recommended value of $G_{N}$,

$$
\begin{aligned}
\alpha_{s} & \cong\left(\frac{m_{e}}{m_{p}}\right)^{12}\left(\frac{\hbar c}{G_{N} m_{p}^{2}}\right) \cong \frac{7.694773265 \times 10^{-12}}{6.67408 \times 10^{-11}} \\
& \cong 0.115293393
\end{aligned}
$$


Quantitatively, independent of the strong coupling constant, it is possible to show that,

$$
\begin{aligned}
G_{F} & \cong\left(\frac{m_{p}}{m_{e}}\right)^{10}\left(\hbar c\left(\frac{2 G_{N} M_{p l}}{c^{2}}\right)^{2}\right) \\
& \cong\left\{\left(\frac{m_{p}}{m_{e}}\right)^{10}\right\}\left(\frac{4 G_{N} \hbar^{2}}{c^{2}}\right) \\
G_{N} & \cong\left(\frac{m_{e}}{m_{p}}\right)^{10}\left(\frac{G_{F} c^{2}}{4 \hbar^{2}}\right)
\end{aligned}
$$

From these relations, we would like to say that,

1) Quantum gravity plays a vital role in weak interactions.

2) By fixing the magnitude of $G_{N}$, from relation (51), magnitude of $G_{F}$ can be estimated and from relation (50), magnitude of $\alpha_{s}$ can also be estimated.

3) By fixing the magnitude of $G_{F}$, from relation (52), magnitude of $G_{N}$ can be estimated and from relation (47), magnitude of $\alpha_{s}$ can also be estimated.

4) With reference to the proposed relations, magnitude of $\alpha_{s}$ seems to be around 0.1153 . The same conclusion can also be extracted from Particle data group's (PDG) review on Quantum chromodynamics [15]. See the following table-3.

Table 3: Magnitude of $\alpha_{s}$ Close To 0.1153

\begin{tabular}{lll}
\hline 1 & $\alpha_{s}\left(M_{Z}^{2}\right)=0.1161_{-0.0048}^{+0.0041}$ \\
\hline 2 & $\alpha_{s}\left(M_{Z}^{2}\right)=0.1151_{-0.0087}^{+0.0093}$ \\
3 & $\alpha_{s}\left(M_{Z}^{2}\right)=0.1148 \pm 0.0014($ exp. $)$ \\
& $\pm 0.0018(P D F)_{-0.0000}^{+0.0050}$ \\
4 & $\alpha_{s}\left(M_{Z}^{2}\right)=0.1134 \pm 0.0011$ \\
5 & $\alpha_{s}\left(M_{Z}^{2}\right)=0.1142_{-0.0023}$ \\
6 & $\alpha_{s}\left(M_{Z}^{2}\right)=0.1151_{-0.0032}^{+0.0033}$ \\
7 & $\alpha_{s}\left(M_{Z}^{2}\right)=0.1158 \pm 0.0035$. \\
8 & $\alpha_{s}\left(M_{Z}^{2}\right)=0.1154 \pm 0.0020$. \\
9 & $\alpha_{s}\left(M_{Z}^{2}\right)$ & $=0.1131_{-0.0022}^{+0.0028}$ \\
10 & $\alpha_{s}\left(M_{Z}^{2}\right)$ & $\cong 0.1156_{-0.0022}^{+0.0021}$ \\
11 & $\alpha_{s}\left(M_{Z}^{2}\right)$ & $\cong 0.1156_{-0.0034}^{+0.0041}$ \\
12 & $\alpha_{s}\left(M_{Z}^{2}\right)$ & $\cong 0.1151_{-0.0087}^{+0.0093}$
\end{tabular}

\section{Discussion and conclusion}

In an advanced and in a semi empirical approach, we proposed peculiar relations (1) to (52). Whether to 'consider them' or 'ignore them', we are leaving the decision to the readers and science community. But it is sure that in final unification point of view, at any 'one' stage of their serious research, one must develop such kind of relations by using which 'gravity' and 'microscopic physics' can be unified. From relations (21) and (32), estimated magnitude of the Newtonian gravitational constant seems to be $(6.66$ to 6.70$) \times 10^{-11} \mathrm{~m}^{3} \cdot \mathrm{kg}^{-1} \cdot \mathrm{sec}^{-2}$.

Considering the wide applicable range of the proposed two assumptions, we are confident to say that, with further research and analysis, 'hidden and left over physics' can easily be explored. In this context, we would also like to stress the fact that, with current understanding of String theory $[1,2]$ qualitatively or quantitatively, one cannot implement the Newtonian gravitational constant in microscopic physics. This 'drawback' can be considered as a characteristic 'inadequacy' of modern unification paradigm. Proceeding further, with reference to String theory models, proposed two pseudo gravitational constants and presented semi empirical relations can be given some consideration in developing a "workable model' of 'final unification'.

\section{Acknowledgements}

One of the authors, Seshavatharam U.V.S, is indebted to professors K.V. Krishna Murthy, Chairman, Institute of Scientific Research in Vedas (I-SERVE), Hyderabad, India and Shri K.V.R.S. Murthy, former scientist IICT (CSIR), Govt. of India, Director, Research and Development, I-SERVE, for their valuable guidance and great support in developing this subject.

\section{References}

[1] Ashoke Sen. Developments in Superstring theory. CERN Document server, hep-ph/9810356 (2009) https://cds.cern.ch/record/368056/files/9810356.pdf. 29.

[2] Edward Witten. What Every Physicist Should Know About String Theory. GR Centennial Celebration, Strings 2015, Bangalore, India. (2105). http://member.ipmu.jp/yuji.tachikawa/stringsmirrors /2015/26-06-2015-Edward-Witten.pdf.

[3] Salam A, Sivaram C. Strong Gravity Approach to QCD and Confinement. Mod. Phys. Lett., A8 (4), 321-326. (1993) http://dx.doi.org/10.1142/S0217732393000325.

[4] U. V. S. Seshavatharam and S. Lakshminarayana.Towards a workable model of final unification. International Journal of Mathematics and Physics 7, No1, 117-130 (2016). ijmph.kaznu.kz/index.php/kaznu/article/.../pdf_10.

[5] U. V. S. Seshavatharam and S. Lakshminarayana. Understanding the basics of final unification with three gravitational constants associated with nuclear, electromagnetic and gravitational interactions. 61st DAE-BRNS Symposium on Nuclear Physics. F40 http://sympnp.org/proceedings/61/F40.pdf.

[6] U. V. S. Seshavatharam and S. Lakshminarayana Understanding nuclear stability and binding energy with nuclear and electromagnetic gravitational constants. 61 st DAE-BRNS Symposium on Nuclear Physics. A136. http://sympnp.org/proceedings/61/A136.pdf.

[7] K.A. Olive et al. (Particle Data Group), Chin. Phys. C, 38, 090001 (2014). http://dx.doi.org/10.1088/1674-1137/38/9/090001.

[8] Chowdhury, P.R. et al. Modified Bethe-Weizsacker mass formula with isotonic shift and new driplines. Mod. Phys. Lett. A20 p.16051618. (2005). http://dx.doi.org/10.1142/S021773230501666X.

[9] W. D. Myers et al. Table of Nuclear Masses according to the 1994 Thomas-Fermi Model. (From nsdssd.lbl.gov).

[10] Srinivasan, G. The Maximum Mass of Neutron Stars. Bulletin of Astronomic Society of India, 30, 523-547. (2002). http://dx.doi.org/10.1007/s001590200016.

[11] Sebastien Guillot et al. Measurement of the Radius of Neutron Stars with High S/N Quiescent Low-mass X-ray Binaries in Globular Clusters. Astrophys.J. 772 (2013). http://dx.doi.org/10.1088/0004 $637 \mathrm{x} / 772 / 1 / 7$.

[12] E. Rutherford, the Scattering of $\alpha$ and $\beta$ rays by Matter and the Structure of the Atom, Philos. Mag., vol 6, ppioi.21, (1911).

[13] Robert Hofstadter, Rudolf Mössbauer. The electron-scattering method and its application to the structure of nuclei and nucleons. Nobel Lecture, (December 11, 1961).

[14] The Periodic Table of the Elements (including Atomic Radius). ww.sciencegeek.net/tables/AtomicRadius.pdf.

[15] S. Bethke, G. Dissertori, and G.P. Salam. Quantum chromodynamics: Olive et al. (PDG), Chin. Phys. C38, 090001 (2014). 